\title{
The Relations of Maturity and Dimensions of Business Intelligence
}

\author{
Rimvydas Skyrius \\ University of Vilnius, Vilnius, Lithuania
}

Rimvydas.skyrius@ef.vu.It

\begin{abstract}
Among developments in the field of business intelligence (BI), the notion of maturity has gained considerable interest for defining the directions to expected value, for summarizing the experience available, and for defining the possible avenues of BI development. There is considerable experience in creating and implementing BI systems, and many gaps separating the declared goals from the achieved results have been discovered. The time distance from the early stages of BI development allows more or less objective evaluation of these stages, while the current state of BI field is often controversial and ill-defined. A set of concepts called BI maturity models have been presented in the academic and professional sources, carrying basically the same mission - to evaluate the experience and extrapolate the future developments. The models, being more or less uniform in assessing the experience, are mostly different in their attitudes towards current state and especially the future (mature) developments of BI, creating controversy in the expectations regarding the future value of BI. The goal of this paper is to define the controversial elements in the existing maturity models, and to suggest a different position on the concept of business intelligence maturity, using most common dimensions of BI features to evaluate maturity expectations.
\end{abstract}

Keywords: business intelligence, business intelligence maturity models, dimensions of business intelligence.

\section{Introduction}

Among developments in the field of business intelligence (BI), the concept of BI maturity has gained considerable interest among researchers and practitioners for defining the directions to expected value, for leveraging the gained experience, and for extrapolating the possible avenues of BI development. With a BI phenomena already being a couple of decades old (if we dismiss an occasional mentioning of the term by H. P. Luhn in 1958), there is considerable experience in creating and implementing BI systems; the achieved results did not always match the declared

Material published as part of this publication, either on-line or in print, is copyrighted by the Informing Science Institute. Permission to make digital or paper copy of part or all of these works for personal or classroom use is granted without fee provided that the copies are not made or distributed for profit or commercial advantage AND that copies 1) bear this notice in full and 2) give the full citation on the first page. It is permissible to abstract these works so long as credit is given. To copy in all other cases or to republish or to post on a server or to redistribute to lists requires specific permission and payment of a fee. Contact Publisher@InformingScience.org to request redistribution permission. goals and many gaps separating them have been discovered.

With BI concept gaining momentum, a number of business intelligence maturity models (BIMM) have been developed that describe a phased structure of business intelligence adoption process. These models have many common features, summarizing the available experience, assessing the current state and de- 
fining directions for future BI developments. While the fact-based experience part of the models is clear and convincing, the projection part that explains vision of mature BI raises a few doubts. This paper looks at the structure and content of the more prominent BIMM, concentrating on their mature stages to specify their common features and possible weaknesses.

The term "maturity model" in the field of information systems is not exactly new, and can be traced back to the year 1973, when Richard Nolan had published his papers on the subject of information systems management in Harvard Business Review and Communications of the ACM (Nolan, 1973; Nolan, 1979). The initial four-stage model encompassed initiation, contagion, control and maturity, and in 1979, two more stages of integration and data administration had been added, expanding the original model to six stages. These stages have been based on the notion that an organization's activities have to improve under technology changes. Nolan's information system maturity model created the basis for other IT-related models to emerge, including business intelligence maturity models. Another maturity model that has played a significant role in development of maturity models for various phenomena in the field of information technologies and systems is Carnegie-Mellon CMM model (Paulk et al, 1995) - a process improvement approach, based on essential elements of efficient processes, that helps to integrate management functions across departmental boundaries and define process improvement goals and guidelines.

The definition of BI maturity is complicated by multi-faceted and often hazy definition of BI itself. Wixom and Watson state that "there is no universally-accepted definition of BI" (Wixom and Watson, 2010). On the other hand, maturity models may assist in identifying strengths and weaknesses of BI application in an organization. As stated in (Lahrmann et al, 2011): "Maturity describes a state of being complete, perfect or ready." The same source presents three maturity concepts:

- the level of knowledge, skills and abilities available for performing business activities;

- the extent to which a specific process is defined, managed, measured, controlled, and effective;

- the respective level of development of a design object.

In the area of BIMM evaluation, researchers and practitioners have compared the available models side-by-side, defining common features (Hribar Rajteric, 2010; Lahrmann et al, 2011; Olszak, 2013) or developing derivative own models (e.g., Shaaban et al 2011). This paper looks at the more prominent BI maturity models, the content of their separate stages and especially the mature stage, and suggests an alternative position regarding the definitions of mature states. The practical dimensions of BI variety that reflect the current state are used to clarify the possible developments of BI maturity.

\section{Analysis of Existing BI Maturity Models}

Further in this paper, an analysis of several different opinions regarding the maturity levels of business intelligence is presented, partly based on (Rebzdaite and Skyrius, 2011). These opinions have been published at different times, and their differences may be explained by different factors being in place at that time. The first presented opinion on BI maturity stages (Deng, 2007) has been announced in 2007, when knowledge management held strong position in the area of information innovations. Here, four maturity levels are specified: data, information, knowledge and wisdom.

At the first, data level of BI maturity, companies collect heterogeneous business data from a variety of sources, cleanse, align and integrate the data. The second level - information level uses integrated data of required quality, and creates meaningful contexts for their presentation. The third level is the level of knowledge. After having received the required information, the users 
make sense of this information on the grounds of available experience, and because of this sense they can estimate causes and notice models that may be applied in business. The fourth BI maturity level is the level of wisdom. It is the highest maturity level that every organization is striving to reach, enabling insightful applications of the knowledge available and changes in the business processes, and business productivity at this level should improve substantially because of timely and efficient decisions.

Another view on BI maturity has been presented in December 2008 by the Gartner Group (Gartner, 2008) in their research report "Gartner's Business Intelligence and Performance Management Maturity Model". It announced a five-stage maturity model which should allow the IT managers, responsible for $\mathrm{BI}$ and general management activities, to have a better understanding of the current stage of BI maturity in the organization. Below, the stages are presented as proposed in (Gartner, 2008).

The first stage is unaware, when the users do not yet realize the true potential of business intelligence. BI use is limited to projects on demand, aiming at satisfaction of individual data analysis needs of the users. The second stage is tactical, or aware - the organizations have already invested into the information needs of certain managers or users for making tactical decisions. However, the other employees do not have this option, and use the old tools for receiving information instead. At this stage it is important to solve problems related to the infrastructure in use, matching data from different systems, data sources that raise doubts in their validity. The third stage is focused, where the managers should cooperate more on the use of business intelligence techniques. At the third stage, the organization is aware of the benefits provided by BI, and has implemented several successful BI projects. However, it is important to implement more projects in different units, thus growing the base of experienced BI users. The fourth stage is strategic. At this stage, the organization is implementing BI strategies on the basis of common strategic goals. $\mathrm{BI}$ initiatives are placed in critical areas of business, creating conditions for BI use to all managers and professionals in the organization. The fifth stage is pervasive. Here the BI systems are integrated into business processes, and they present the required and reliable information to the users when changes happen. The implemented metrics are not only uniform throughout the organization, but are open to the clients and suppliers, thus reinforcing business relations. The main goal of the fifth stage is to maintain leadership even when major events occur, such as mergers, acquisitions or changes in management.

One more example of BI maturity explanation has been presented by Rob Walker in a paper named "The Evolution and Future of Business Intelligence" (Walker, 2009). Here, five levels of BI maturity are presented; however, the outlook is much more ambitious and futuristic.

The first level includes reports, produced with certain latency after the facts happen. Multidimensional OLAP techniques are used; much of the information production is automated.

The second level pretty much resembles the first level; a real-time data analysis options are added, providing the possibility to monitor current situation.

The third level provides real-time actionable intelligence, enabling swift responses to important changes in the environment. Walker points out, however, that "a company is not a fly-by-wire jet fighter", which goes very much in line with what has been presented earlier in this paper regarding intelligence velocity - the businesses that require real-time intelligence and real-time action are not that numerous.

Two more levels are proposed: according to Walker, the fourth level is already in existence, and the fifth level is about to come in the near future. Being at the fourth level, a company is enabled to test selected strategies before implementing them, simulating scenarios. The fifth level achieves a resemblance to "fly-by-wire" principle: by using artificial intelligence as well as tradi- 
tional statistical analysis, business parameters can be optimized immediately. In other words, while fourth level would leave space for user judgment, the fifth level would perform automatic optimization of business processes.

The Data Warehousing Institute's (TDWI) BIMM is focused mostly on data organization aspect, and covers six stages (Eckerson, 2007). The prenatal stage essentially conforms to the mission of regular management information systems with fact-based and predominantly financial reporting. The infant stage features local efforts by analysts to gain insights, mostly based on so-called spreadmarts. The child stage uses data marts, or single-area data warehouses, interactive analytical reports, and serves more complex questions of the "Why did it happen?" type. The teenager stage features data warehouses of larger scale, due to the data integration efforts, and accentuate the monitoring function. The adult stage signals a transition from division level solutions to enterprise-wide data warehouses; this transition has to overcome divisional politics and implement consistency standards. The final, sage stage, features federated development that reacts swiftly to dynamic needs; blended applications that embed analytics into core activities; decision automation incorporating rules and models; and commercialized services providing intelligence products to the outside world.

An Enterprise Business Intelligence Maturity (EBIM) model, proposed by Tan et al (Tan, Sim, Yeoh, 2011), comprises five stages. The first stage is initial, where no organized BI pattern exists. The second stage is repeatable, and features some centrally imposed standards, like master data model, and generally growing interest in BI. The third stage is defined, and its most characteristic feature is centralization and company-wide approach to BI. The fourth stage is managed, where, in addition to centralization of data-related procedures, the maintenance of business rules is also centralized, although their processing is distributed. The final, fifth stage is optimizing, where the unified infrastructure fades into background, and analytical functions are provided as services.

Table 1 contains the descriptions of mature stages of the maturity models discussed above.

Table 1. The content of the mature stages of discussed BI maturity models

\begin{tabular}{|l|l|}
\hline Model / source & Description of mature stage \\
\hline BI Maturity Hierarchy (Deng, 2007) & $\begin{array}{l}\text { Wisdom: sound, timely and efficient business } \\
\text { decisions. }\end{array}$ \\
\hline $\begin{array}{l}\text { Gartner Maturity Model for Business } \\
\text { agement (Gartner, 2008) }\end{array}$ & $\begin{array}{l}\text { Pervasive: BI and PM (performance measure- } \\
\text { ment) become pervasive across all areas of busi- } \\
\text { ness and across part of corporate culture; flexi- } \\
\text { bility to adapt to changes. }\end{array}$ \\
\hline $\begin{array}{l}\text { Walker's BI maturity model (Walker, } \\
\text { 2009) }\end{array}$ & $\begin{array}{l}\text { The fifth level achieves a resemblance to "fly- } \\
\text { by-wire" principle: by using artificial intelli- } \\
\text { gence as well as traditional statistical analysis, } \\
\text { business parameters can be optimized immedi- } \\
\text { ately. }\end{array}$ \\
\hline TDWI BIMM (Eckerson, 2007) & $\begin{array}{l}\text { Sage stage: mix of centralized (EDW) and de- } \\
\text { centralized (development, custom solutions) el- } \\
\text { ements; Centers of Excellence. }\end{array}$ \\
\hline $\begin{array}{l}\text { Tan, Sim and Yeoh Enterprise Busi- } \\
\text { ness Intelligence Maturity model (Tan, } \\
\text { Sim, Yeoh, 2011) }\end{array}$ & $\begin{array}{l}\text { Optimizing stage: single view of truth, enter- } \\
\text { prise data convergence, analytical services and } \\
\text { analytical competitor. }\end{array}$ \\
\hline
\end{tabular}


All of the above descriptions of the mature stages lean towards ideal organization, automation of intelligence processes and implementation of "once and for all" model of near-perfect BI. The mature stage is seen as an ideal state of BI, with automation and optimization of key business activities in real time. Regarding automation, the authors of the above models are not exactly original - the idea of automating human intelligence by sophisticated programming has been around for a long time; we can recall the history of expert systems and related expectations. The limitations of such approaches have been presented way back in 1994 by one of the authorities in management, Henry Mintzberg, when writing about the attempts to automate the processes of strategy development and stating that all attempts to program them have brought no more success in imitating intuition than psychoanalysis methods enabled the simulation of empathy (Mintzberg, 1994). To author's opinion, a much more realistic case would be the development of sustainable principal intelligence competencies that would be flexible, adaptable to changes in environment and adequately supported by IT tools. These competencies should be based on balanced expectations regarding the BI maturity states, and, to author's opinion, one of the possible ways of doing this would be the assessment of current variety of BI forms, reflected in practical dimensions. Based on existing research, including the author's former work in the area, the following section examines the most common dimensions of business intelligence to define the possible measures of maturity.

\section{The Dimensions of $\mathrm{BI}$ and Their Relation to Maturity}

The current experience with BI systems points out to several principal dimensions that might provide a more realistic approach to BI maturity. The dimensions examined below are: internal and external orientation, centralization and decentralization, functional scope, question complexity, task automation, initiative and velocity.

\section{Internal and External Orientation.}

The monitoring of internal and external business environment has significant differences:

- internal - based on reliable own information and clarity; nearly automatic data integration under internal set of rules; source data supplied by internal information systems whose behavior, product and logic are known, understandable and under own single control;

- external - directed towards detection of weak external signals and making sense thereof; the true value of data is discovered only by experimenting or actual use; information is integrated by soft rules, middleware, passive methods; IT support is chaotic and of limited flexibility.

Although the processes and information sources for internal and external orientation are different, they do not imply the existence of two separate intelligence fields. The very term "intelligence" presumes the level of understanding that is comprehensive and supported by possession of varied, multidimensional and mutually supportive information. The division of BI into internal and external, or separation of BI and competitive intelligence should be considered immature; and the mature stage should unconditionally cover both internal and external information. In other words, the required coverage of business environment is to be supported by appropriate mix of internal and external intelligence, providing a richness of context that adequately serves the intelligence needs. For decision makers that have to be aware of both internal and external context, a mature BI system should possess certain agility to allow easy concentration on any issue of interest, regardless of its location. 


\section{Centralized and Decentralized BI.}

The dimension of centralization should relate to the nature of business - uniform or diverse, centralized or distributed. Various approaches exist, based on sometimes contradictory arguments: centralized BI systems seem to be favored less because of their inertia and low flexibility, although such system has more potential for necessary transparency and presentation of "big picture". Centralization usually covers infrastructure and standards; democratic and federated approaches support the decentralized part. Too many standards impair flexibility, while too few standards create challenges to integration. Lately many votes are given for self-service BI; however, it supports the emergence of isolated "intelligence islands" and shadow systems. A longlasting use of spreadsheets, or so-called Excelization, is a proof to this. Many published cases on company-wide BI solutions (e.g., Fogli, Guida, 2013; Iş1k, Jones, Sidorova, 2013; Popovič et al, 2012; Zimmer, Baars, Kemper, 2012) stress the necessity to achieve a balanced mix of centrally managed standards on one side, and flexibility for special needs of business users on another. A mature BI should have a balance of centralized and decentralized approaches, based on horizontal propagation of information and appropriate intelligence culture.

\section{Functional Scope of BI Applications}

The functional scope of BI applications ranges from simple information presentation by reporting and graphing to advanced and flexible features - sophisticated search engines, data and information integration, performance management, predictive analytics, data and text mining, and others. These applications should be available across different technology platforms, including mobile and cloud-based implementations. On one hand, the set of BI tools should be adequate to match the problems that would emerge; on the other hand, the presence of a large and sophisticated toolset might be confusing to the users who, for most of the time, would require just a fraction of this toolset. A tiered approach has been proposed earlier by the author (Skyrius, 2008) to balance functional richness ("just in case") with what is needed most of the time. This approach would employ the split of functional support into close tier (single) and distant tiers (single or multiple). The close tier would contain the simple support environment elements that are required most of the time and can be easily configured:

- the most important data to be watched - sales, orders, cash-at-hand, market share;

- information search tools - internal queries in databases and text bases; simple search in public sources; tools to arrange search results - sorting of results, classification, annotation etc.;

- simple processing tools: templates, financial models, other simple analysis and modeling tools.

The second tier would contain the more complex support elements that are required less often, and might include:

- remote and complex information sources with advanced search tools;

- assorted information integration tools;

- modeling tools for forecasting, regression, simulation;

- data analysis technologies - multidimensional OLAP environments, data and text mining software;

- presentation, reporting, graphing and visualization tools.

In the area of BI functionality, some controversy is created by a conflict of the terms "business intelligence" and "business analytics". Their separation is usually based on the time perspective: $\mathrm{BI}$ is understood as past-facing, while business analytics are future-oriented. The author sees this 
as a problem of an agreed use of a term, but at the same time several issues come to mind. Just like in the case of internal and external intelligence, or any kind of intelligence for that matter, analytics play a key part in the intelligence process, and regardless of whatever meaning is assigned to the terms, they are parts of an integral whole. And similar to the balance of internal and external BI orientation, the separation of BI and business analytics seems artificial and immature.

\section{Question complexity}

Question complexity deals with the range of intelligence questions from simple questions involving few information sources and procedures to complicated informing questions, requiring integration of diverse information from a number of sources, a wide set of functions and usually a number of human participants in the process. The tiered approach should provide a base set of support tools that would create a starting position in solving business problems, and allow simple updates when required, thus adding its share to system agility. The analysis of various dimensions of information needs shows that, as the complexity and uncertainty of problems grow, the potential of efficient IT use to automate informing functions diminishes, while the requirements for support flexibility and variety increase. We can look at a complexity scale of possible BI questions that are presented in many sources; the example below is a generalized list of such questions by growing complexity:

- What has happened?

- Why did it happen?

- Where is the problem?

- What action is needed?

- What are the expected outcomes?

- What may happen next?

- What's the best we can do?

The first three questions represent the simple part of the question complexity spectrum and can largely be handled automatically by appropriate software. According to many sources on BIMM, mature BI is supposed to provide automatic answers to complex questions. However, with increasing question complexity, automation issues get complicated and require participation from human users in terms of selection of alternatives, choices, assumptions, where heuristics and efficient access and use of support environment play the principal role. In this case, mature BI should provide the necessary flexibility, and it should not impose its own complexity on question complexity.

\section{Task automation.}

Regarding BI task automation, we can clearly separate tasks that are easy to automate (database querying, Web search), and hard-to-automate human, or heuristic tasks (sense making, semantic integration). In the middle there is a "gray area" where tasks experience attempts to be automated, but due to uncertainty and low repeatability human initiative or guidance are required (data integration, influence modeling, rule creation and use). If we group the various tasks in information activities by their automation potential, this criteria reflecting the routine or non-routine nature and other features defining required efficiency or flexibility, we can roughly fit them into three groups (Skyrius, 2014):

1. Examples of easily automated tasks:

- database querying,

- Web search,

- arranging and combining data, 
- recommenders.

2. Examples of human tasks mostly based on heuristics:

- semantic integration,

- sense-making,

- causal analysis,

- creation of alternatives,

- negotiations.

3. Examples of "gray area", where sophisticated support and synergy between IT and user are sought:

- integration of data - manual or automatic;

- influence modeling;

- rule creation and use;

- text analytics;

- simulation, "what-if" modeling.

Of the three groups, the third one is the most interesting and ill-defined in terms of possible synergy between IT and users. Regarding the definition of this "gray area", there are some vague lines between automation and human action: for instance, we can fully automate a simulation experiment, or we can leave all emerging "what-if" questions to be handled by human actors. Similarly, customer behavior rules can be induced by the data mining software, or they can be inferred by analysts interpreting the results of analysis. The mature BI might be expected to have automated routine functions, and some of the "gray area" functions with bypass capabilities if required to switch to manual mode.

\section{Initiative}

The initiative dimension is located between user-driven (or question-driven) and data-driven intelligence. While user-driven BI performs user pull and usually deals with a business problem or an assumption, data-driven BI performs system push and relies on pre-built models and applications. Currently, the term of "data-driven business intelligence" is frequently used, especially with emergence of big data and unstructured information. It is interesting to note that many published cases, having been presented like data-driven BI, actually have a business question present in the first place.

User-driven BI procedures are initiated by a business question, usually followed by a check on availability of support resources, and then by a search for cues or patterns that would make the required sense. A side issue of user-driven BI is self-service BI and analytics, whose roots firmly rest in the use of spreadsheets. While self-service BI encourages users to perform independent information services themselves, and base decisions on data instead of intuition, it creates the risk of chaotic reporting within an organization. To reduce this risk, key metrics and a unified business vocabulary should be determined together with processes for creating intelligence reports and publishing them.

While data-driven $\mathrm{BI}$ is one of the favorite features in the available BI maturity models, it tends to preempt the users, reducing flexibility and the virtues of human actor. At the same time, alerts and other cases of automated informing in proactive systems belong to a data-driven approach as well; there are numerous examples of data-driven intelligent functions: complex alerts, flexible 
inventory management, adaptive scheduling and others. These functions are of regular nature and can form a basis or a part of monitoring system; this should mostly apply to regular data from operations regarding internal environment and close external environment (customers, suppliers, partners, competition).

In a mature BI system, a balance of both approaches could be presumed. The use of a two-tier support structure, mentioned earlier in this paper, might assist in this balance in a way that common needs, served by repeating routine data-driven procedures, are mostly satisfied in the $1^{\text {st }}$ tier of this environment, with a probable exception of common complex needs - e.g., discovery of hidden rules in big data or monitoring the environment for emerging innovations. The emergence of special needs, requiring additional attention and user involvement, might require more support resources than $1^{\text {st }}$ tier can provide, and thus facilitate the use of the additional resources found on $2^{\text {nd }}$ tier or further tiers, if they do exist.

\section{Velocity}

The velocity dimension distinguishes between real-time and right-time BI. Quite a few BI maturity models accentuate real-time BI as an imminent feature of mature stage. However, an earlier survey, performed by the author (Skyrius and Bujauskas, 2011) has shown that for a surveyed sample of 203 participants, representing mostly small and medium business managers, the most common latency of informing appeared to be one day, showing that the urgency of information presentation in most cases is defined by the real business velocity, and a mature BI system should be able to adapt to the variations in this velocity.

\section{Conclusions}

The analysis of the BI dimensions has shown that, based on current experience of BI use, a more balanced view on the concept of BI maturity is required to counter the often-inflated expectations and to survive inevitable changes in the future. All BI maturity models presented above, as well as most of the other BI maturity models, have the same mission - to evaluate the accumulated expertise in the area, assess current situation, estimate a trend and extrapolate it towards some ideal state. If evaluated along these guidelines, maturity represents a fact, or a point in time where the BI system reaches its goals driven by expectations. The most common features of the maturity models are automation of high-level information activities, including automatic optimization; real-time reaction to changes in the environment; rigid centralized standards. However, features required in a dynamic business environment, but omitted in most of the models, are flexibility, agility, ability to adapt while maintaining core competencies.

For BI maturity models to become more realistic, especially regarding their final phases, more realistic criteria are required. If the goal is to optimize decisions, a problem of choosing optimization criteria emerges: are the criteria just internal, if BI addresses internal processes, or external criteria are used as well? The use of external criteria renders optimization impossible because of changing context. For this reason the users of BI have to make up their mind whether they are after optimal decisions or just good enough decisions, whose search requires less time and effort. Here we can quote Snowden and Boone: "In a chaotic context, searching for right answers would be pointless" (Snowden and Boone, 2007). Information technologies can adapt to changing situations and be programmed to work flawlessly in a certain area, but in unknown environment that programmers have not encountered before, IT won't be able to function appropriately. A good point is provided by Evelson: "while earlier-generation BI technologies have matured into industrial-strength solutions, they have largely failed to address one simple, pragmatic business reality: the need for flexibility and agility. As a result, centralized BI environments are anything but agile" (Evelson, 2011). 
The routine part of BI technologies might just mean an information system doing a good job. Whatever practice becomes routinized, it operates as a stable part of BI, but other changes will come and require new practices; the ability to accept them might be the important part of the idea of maturity proposed in this paper. Intelligence is cyclical as any process of perception, so the maturity cycle can be viewed as a sequence of waves of local maturity. Moreover, history of maturity models (not only for BI) shows that often, for a given area of activity, they signal a coming of profound changes whose time has come.

We can make an assumption that BI as a fluid and ever-changing phenomenon will never be mature in a sense of stability and routine; then the very idea of BI maturity may be questioned. The other option to define a concept of BI maturity might be a review of a separate technology for its maturity life cycle: some technologies emerge, gain adoption and experience, become established and routinized, or fade away and are replaced by other emerging technologies that are at the beginning of their maturity cycle.

Based on aforesaid, the required features of future BI instruments would be:

- flexible support for human analytical and decision support functions instead of attempts to perform these functions automatically;

- ability to perform flexible analysis of huge data volumes;

- ease and convenience of use; the technology should "melt" against the background of a problem, thus freeing user attention for the heart of the problem;

- abilities to adapt to changing environment by providing required agility and vitality by compact set of standards, flexible "federated" development, recurring evaluation of information needs;

- development and support of analytical culture based on unrestricted communication, exchange of information between participants of intelligence processes, thus maintaining an intelligence community in an organization.

\section{References}

Deng, R. (2007). Business intelligence maturity hierarchy: A new perspective from knowledge management. Available at: http://www.information-management.com/infodirect/20070323/1079089-1.html

Eckerson, W. (2007). Beyond the basics: Accelerating BI maturity. The Data Warehousing Institute Research. Available at: http://download.101com.com/pub/tdwi/Files/SAP_monograph_0407.pdf

Evelson, B. (2011). Buyer's guide: How agility will shape the future of business intelligence. Available at: http://www.computerweekly.com/feature/Buyers-Guide-How-agility-will-shape-the-future-ofbusiness-intelligence

Fogli, D., \& Guida, G. (2013). Knowledge-centered design of decision support systems for emergency management. Decision Support Systems, 55, 336-347.

Gartner's Business Intelligence and Performance Management Maturity Model. (2008). Available at: http://www.gartner.com/DisplayDocument?id=500007

Hribar Rajteric, I. (2010). Overview of business intelligence maturity models. Management, 15(1), 47-67.

Işık, Ö., Jones, M. C., \& Sidorova, A. (2013). Business intelligence success: The roles of BI capabilities and decision environments. Information \& Management, 50, 13-23.

Lahrmann, G., Marx, F., Winter, R., \& Wortman F. (2011). Business intelligence maturity: Development and evaluation of a theoretical model. Proceedings of the $44^{\text {th }}$ Hawaii International Conference on System Sciences, 1-10.

Mintzberg, H. (1994). The rise and fall of strategic planning. New York, NY: The Free Press.

Nolan, R. (1979). Managing the crisis in data processing. Harvard Business Review, 57(2), 115-126. 
Nolan, R. (1973). Managing the computer resource: A stage hypothesis. Communications of the ACM, 16(4), 399-405.

Olszak, C. (2013). Assessment of business intelligence maturity in the selected organizations. Proceedings of the 2013 Federated Conference on Computer Science and Information Systems. Krakow, Poland, September 8-11, 2013. 951-958.

Paulk, M., Weber, C. V., Curtis, B., \& Chrissis, M. B. (1995). The capability maturity model: Guidelines for improving the software process. SEI series in software engineering. Reading, Mass.: AddisonWesley.

Popovič, A., Hackney, R., Coelho, P. S., \& Jaklič J. (2012). Towards business intelligence systems success: Effects of maturity and culture on analytical decision making. Decision Support Systems, 54, 729-739.

Rebzdaite, V., \& Skyrius, R. (2011). Business intelligence maturity and prospects. Changes in Economy and Property Value: Trends and Management Techniques. Conference proceedings. Vilnius, Lithuania: Vilnius University, March 25, 2011. 136-145.

Shaaban, E., Helmy, Y., Khedr, A., \& Nasr, M. (2011). Business intelligence maturity models: Toward new integrated model. The International Arab Conference on Information Technology (ACIT'11), Riyadh, Saudi Arabia, Dec. 11-14, 2011.

Skyrius, R. (2008). The current state of decision support in Lithuanian business. Information Research, 13(2), paper 345. Available at: http://InformationR.net/ir/13-2/paper345.html

Skyrius, R. (2014). The split of information needs support between the users and information technology. DSS 2.0 - Supporting Decision Making with New Technologies: 17th conference for IFIP WG8.3 DSS, 2-5 June 2014, Paris, France : supplemental proceedings / Editors : Gloria Phillips-Wren, Sven Carlsson. IOS Press, 227-238.

Skyrius, R., \& Bujauskas V. (2011). Business intelligence and competitive intelligence: Separate activities or parts of integrated process? The Global Business, Economics and Finance Research Conference. London, UK, July 2011.

Snowden, D., Boone, M. A. (2007). Leader's Framework for Decision Making. Harvard Business Review, November, 69-76.

Tan, C.-S., Sim, Y.-W., \& Yeoh, W. (2011). A maturity model of enterprise business intelligence. Communications of the IBIMA, 2011, Article ID 417812.

Walker. R. (2009). The evolution and future of business intelligence. Available at: http://www.information-management.com/infodirect/2009 140/business intelligence bi-10016145$\underline{1 . h t m l}$

Wixom, B. H., \& Watson, H. J. (2010). The BI-based organization. International Journal of Business Intelligence Research, 1(1), 13-28.

Zimmer, M., Baars, H., \& Kemper, H.-G. (2012). The impact of agility requirements of business intelligence architectures. HICSS 2012: Proceedings of the $45^{\text {th }}$ Hawaii International Conference on System Sciences, 4189-4198. 


\section{Biography}

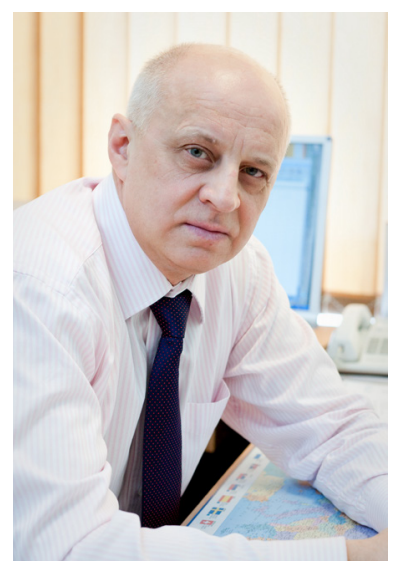

Rimvydas Skyrius is a Professor and head of the Economic Informatics department at the University of Vilnius, Lithuania. He received his doctorate in Operations Research and Computer Applications from ASU-Moscow Institute in 1986, and his Master's degree from the University of Vilnius in 1978. His principal research areas are IT-based decision support in business and management, business intelligence and management information needs, and he has published a monograph, a number of articles and conference papers on the subject, as well as co-authored several textbooks in the field. He is also a member of Editorial board of Economics and Organizations and Markets journals, published by the University of Vilnius, Lithuania. 